\section{LA EDUCACIÓN FÍSICA EN CHILE: UN ANÁLISIS DE LAS CREENCIAS DEL PROFESORADO DE LA ENSEÑANZA PRIMARIA Y SECUNDARIA}

\author{
PHYSICAL EDUCATION IN CHILE: AN ANALYSIS OF PRIMARY AND \\ SECONDARY EDUCATION TEACHERS'BELIEFS
}

\author{
EDUCAÇÃO FÍSICA NO CHILE: UMA ANÁLISE DAS CRENÇAS DOS \\ PROFESSORES DO PRIMÁRIO E ENSINO MÉDIO
}

\begin{abstract}
Alberto Moreno-Doña*, Enrique Rivera-García**, Carmen Trigueros-Cervantes ${ }^{\star \star *}$
\end{abstract}
Resumen: Se analizan, cualitativamente, las creencias de los docentes de educación física respecto a la función social que debiera cumplir la asignatura. En dicho análisis se identifican barreras personales, profesionales e institucionales encontradas en dicha tarea y las soluciones que ellos proponen al respecto. Se realizaron tres grupos focales y doce entrevistas en profundidad. Los procedimientos de análisis empleados, en general se ciñeron a las propuestas de fragmentación y articulación de la "Grounded Theory". Se concluye que existe una relación dicotómica para algunos, complementaria para otros, entre una EF que reproduce una perspectiva tradicional ligada al deporte, el desarrollo de la condición física y ciertos estereotipos sociales; y otra visión que entiende que la educación física sirve para desarrollar competencias de comprensión y transformación de las desigualdades sociales del alumnado.

\section{Keywords}

Primary and

Secondary

Education.

Professional role.

Social responsibility.

Abstract: This paper uses qualitative methods to analyze physical education teachers' beliefs with regards to the social role that the discipline should perform. It identifies personal, professional and institutional barriers to achieving this social role and considers proposals from the teachers to overcome these barriers. Three focus groups were created and 12 in-depth interviews were carried out. The methods of analysis employed were anchored in the perspectives of fragmentation and articulation, as expressed in "grounded theory". The paper concludes that, for some teachers, a dichotomic relationship exists between a physical education that reproduces a traditional approach (linked to sports and physical condition) and some specific social stereotypes, while for other teachers this relationship is a complementary one. There is another perspective that suggests that physical education can help develop the ability among students to comprehend and transform social inequalities.

Palavras-chave

Ensino fundamental e médio.

Papel profissional. Responsabilidade social. profissionais e institucionais enfrentadas nesta tarefa e as soluções que propõem so-
Resumo: Analisamos, qualitativamente, as crenças dos professores de Educação Física sobre a função social que deve cumprir a disciplina, identificando barreiras pessoais, bre isso. Foram realizados três grupos focais e doze entrevistas em profundidade. Os métodos de análise utilizados, em geral, foram baseados na proposta de fragmentação e articulação de "Grounded Theory". Conclui-se que há uma relação dicotômica para alguns, e complementária para outros, incluindo uma EF que reproduz uma perspectiva tradicional ligada ao desporto, o desenvolvimento da aptidão física e certos estereótipos sociais; e outro ponto de vista que entende que a Educação Física é desenvolver habilidades de compreensão e transformação das desigualdades sociais nos alunos.
*Escuela de Educación Física. Facultad de Filosofía y Educación. Pontificia Universidad Católica de Valparaíso. Valparaíso, Chile.E-mail: alberto.moreno@ucv.cl

** Facultad de Ciencias de la Educación. Universidad de Granada. España. E-mail: erivera@ugr.es

*** Facultad de Ciencias de la Educación. Universidad de Granada. España. E-mail: ctriguer@ugr.es

Recebido em: 24-06-2014 Aprovado em: 12-09-2014 (c) (1) () Licence 


\section{INTRODUCCIÓN}

Hemos pasado de la controversia (poder dialogar y enfrentar posiciones) a la crisis (asumir, que no aceptar, las imposiciones que nos vienen desde los que ejercen el poder que otorga la mayoría democrática), del cuestionamiento constante de nuestro hacer pedagógico a aceptar todo lo que nos viene desde el currículum nacional como lo único que se puede enseñar en la escuela. Asumimos que la cultura social debe ser transferida a la cultura escolar y, por ende, vemos como el pensamiento neoliberal imperante, la economía de mercado, los medios de comunicación de masas puestos al servicio de los gobiernos, van haciendo lentamente su trabajo y logrando que los ciudadanos vayan asumiendo su discurso e ideología como realidad inevitable; de aquí al pensamiento único, tal como nos adelantaba Orwell (1949), o al mundo feliz de Huxley (1932) solo queda un escalón. Estamos asistiendo al abandono progresivo de los aspectos sociales y valóricos de la educación, rindiéndonos a su mercantilización desde la reproducción de los principios que lo rigen (BALL; 2004; EVANS, 2013). En este contexto, la Educación Física (EF) no ha quedado excluida del control directo o indirecto que ejerce el poder en cualquiera de sus formas, vemos que, aunque con discursos contradictorios en muchas ocasiones, al final lo que se impone es una cultura social acrítica, sesgada y empobrecida interesadamente por el poder desde los medios de comunicación para adormecer a los ciudadanos.

Centrándonos en el caso chileno y entrando en la nueva propuesta curricular del área de $\mathrm{EF}^{1}$, podemos ver que lo que mencionábamos en el párrafo anterior comienza a cobrar visos de realidad. La primera medida que se adopta es un cambio de nombre, pasando a ser llamada "Educación Física y Salud" (EFYS). Ante esta nueva denominación nos cabe abrir dos interrogantes: ¿La EF que se venía desarrollando en los centros educativos no era saludable? y ¿qué concepto de salud subyace en esta nueva denominación? En segundo lugar reconoce a la EFYS como una "asignatura central de la educación escolar, que se enmarca en el proceso de formación integral del ser humano". Ante esta afirmación nos preguntamos si la línea de esta "nueva" asignatura va a caminar a partir de ahora por el respeto de las inteligencias múltiples (GADNER, 1994; 2005), o al menos tendrá presente la inteligencia emocional (GOLEMAN; 1996), buscando la globalidad desde la focalización de la atención en aquellas que más directamente se relacionan con la materia (corporal y cinestésica, intrapersonal, interpersonal y naturalista). Pero la duda queda resuelta a partir del siguiente punto y seguido, donde una vez más se vuelve al "modelo" y se propone que esto será posible solo si se produce "práctica regular de actividad física" a través de la cual "los estudiantes podrán desarrollar habilidades motrices, actitudes proclives al juego limpio, el liderazgo y el autocuidado". Atención al nuevo concepto que se introduce: "liderazgo", en principio, en una lectura rápida, puede parecer inocuo, pero no creemos que esa sea la intención. En la trastienda del concepto se identifica claramente el abrazo de la EFS al paradigma darwinista (BARBERO; 2012), donde la perfomance cobra todo su sentido en cada uno de los "énfasis temáticos" que le acompañan (la importancia del movimiento, el desarrollo de la condición física, las cualidades expresivas, la iniciación deportiva, conjunción de factores para una vida activa, seguir las reglas del juego y cooperación y trabajo en equipo); conformando la estructura básica del modelo de EFS que debe presidir la enseñanza básica chilena. Si todo discurre por los cauces establecidos es posible que al final nos encontremos a un pre-adolescente con un "estilo de vida activo y saludable" que le va a garantizar "múltiples beneficios individuales y sociales".

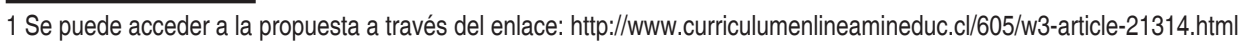


Sobre el papel se apuesta por una formación integral de la persona, pero la realidad dista mucho de las intenciones. La obsesión por la visibilización del logro (PÉREZ; SOTO, 2011) desde el "accountability" (rendimiento de cuentas) a través del Sistema de Medición de la Calidad Educativa (SIMCE) que ahora ha llegado también al área de EF, hace que la discriminación en base a la competencia motriz y el Índice de Masa Corporal (IMC) se haga evidente (MORENO et al. 2013). El poder, a través de los medios de comunicación nos transmite una imagen corporal que dista mucho de la realidad de nuestros escolares, especialmente de aquellos socialmente menos favorecidos. Nos ofrecen el éxito deportivo y el cuerpo perfecto como referentes a lograr, a sabiendas de que solo unos pocos podrán ocupar su sitio en el olimpo de los dioses. Como plantea Evans (2013), solo se admiten los extremos; muestra competencia motriz o por el contrario serás calificado como torpe, gordo o delgado; no existe el término medio en el segmento de la performance. Esta presión que directamente sufre el estudiante es producida por el sistema desde la pedagogía del biopoder, transformando en normatividad su propia percepción de cuerpo saludable asociado a la delgadez (HARWOOD; 2009). Pero no olvidemos que es el docente quien queda responsabilizado de su ejecución; es él quien debe lograr que sus alumnos alcancen la excelencia, ya que si el producto final no cumple con los estándares marcados y de sus clases salen niños gordos e inactivos, con bajos niveles de rendimiento deportivo, será identificado como el principal causante del fracaso y quién sabe si el motivo de su depuración.

Finalmente, lo que nos queda es el mismo modelo de EF que se lleva trabajando en las escuelas desde hace más de 20 años. Una EF que sigue pivotando en torno a dos ejes: la condición física y el deporte. Desde el primero se busca el cuerpo delgado, desde la erradicación de la plaga del siglo XXI: la obesidad. El problema se encuentra en el cómo y el quién. A la primera cuestión la solución que se aporta es optar, una vez más, por el modelo biomotriz, obviando que el concepto de salud es mucho más amplio, que debe traspasar los límites del cuerpo orgánico para transitar en lo inter e intrapersonal en un contexto medioambiental adecuado (DEVÍS, 2000). Respecto a la segunda cuestión, quién o quiénes actuarán de vanguardia en la batalla, son los profesores de EF quienes, gracias al aumento a cuatro horas de clase, deben obrar el milagro de transformar la grasa en músculo y la obesidad en delgadez. El segundo pivote, una vez más lo ejercerá el deporte, que actuando como panacea sanadora de todos los males logrará un doble objetivo: crear el semillero que nos visibilice en el mundo desde el logro de la excelencia deportiva y facilitar un medio adecuado al resto de personas para que disfruten consumiendo ocio activo.

Frente a este modelo de EFYS existe otra forma de entender la EF más cercana a enfoques críticos. Podemos revisar el excelente trabajo realizado por Devís (2006; 2012), en el que se realiza una amplia revisión de las perspectivas socio críticas en EF a nivel mundial. Pero si reparamos en el análisis que ofrece el autor, podemos ver que la mayoría de ellas se posicionan en lo que acertadamente identifica Vicente (2013), como miradas críticas a la EF más que enfoques situados en los principios de la Pedagogía Crítica. Compartimos su discurso, pero sin olvidar que se realiza desde una mirada evaluativa externa al modelo.

En la misma línea que Tinnig (2002) citado por Sicilia-Camacho y Fernández-Balboa (2009), pensamos que es el momento de replantearnos seriamente hacia dónde debiera caminar una EF crítica, y si es preciso renunciar a asumir un papel protagónico en la liberalización del otro para centrarnos en facilitar a los estudiantes el encuentro con ellos mismos, para que, desde el análisis crítico y la reflexión, construyan sus propias decisiones y actuaciones frente a los modelos sociales dominantes (SICILIA; FERNÁNDEZ, 2009). 
Lo que sí entendemos como imprescindible es la necesidad de salir de la investigación contemplativa y pasar a modelos de acción. Evidentemente habrá que vencer resistencias, tal y como plantea Lemos et. al. (2012). Las críticas al modelo dominante están realizadas, lo que es necesario es ofrecer otras formas de hacer en el aula de EF que convenzan e ilusionen a los profesionales. Alternativas reales y posibles, es muy probable que tengamos que renunciar a la igualdad de oportunidades que puede ser el espejismo que nos impida ver la realidad de la brecha social y apostar por una igualdad de posiciones (DUBET, 2012) en la que se produzca una redistribución de la riqueza que facilite a todos los ciudadanos el acceso a unas condiciones de vida dignas, una educación de calidad, seguridad y servicios básicos. En esta dirección se está empezando a mover la EF, apareciendo alternativas al modelo descrito inicialmente, que buscan prácticas transformadoras que enriquezcan a los estudiantes (LORENTE; JOVEN, 2009; PRAT; SOLER, 2009; SOLER, 2009; TIRADO; VENTURA, 2009). Más conectadas con la pedagogía crítica, las propuestas se centran especialmente en la formación del profesorado de EF, pero sin una clara conexión con el aula básica y media (LORENTE; KIRK, 2014; MORENO; TRIGUEROS; RIVERA, 2013; MATTA; RICHARDS, en prensa 2; SPAAIJ; JEANES, 2013). Por último, destacar la línea de trabajo en la dirección del Aprendizaje-Servicio en EF, que puede ser una potente herramienta para llevar la transformación social de las aulas universitarias a contextos escolares y sociales de escasos recursos o en situación de riesgo social. En esta línea se puede revisar el trabajo de Cervantes y Meaney (2013), que realizan una exhaustiva revisión sobre el tema. También en nuestro contexto podemos destacar los trabajos de Rubio, Campo y Sebastiani (2014) y Boneta (2014). Desde una perspectiva más práctica podemos revisar el trabajo de Chiva, Gil y Hernando (2014) que nos ofrecen una propuesta desde la Expresión Corporal y los Juegos Motrices.

Desde la revisión realizada de las dos caras de la EF tomando como referente la propuesta curricular chilena, perfectamente trasladable a otros contextos, queda claro que estamos asistiendo a un momento crítico. La potencia del modelo neoliberal cada día presiona más para imponer unas bases para el área bajo parámetros de objetividad, rendimiento y visibilidad, invalidando todo aquello que no cumpla lo establecido. Ante esta realidad, es necesario desarrollar una actitud partisana que defienda una EF crítica pero desde la acción, ofreciendo alternativas reales que transformen a nuestros alumnos en mejores ciudadanos. La investigación que se presenta quiere tomar el pulso a los docentes y valorar en qué estado de fuerzas nos encontramos. Para ello, nos hemos centrado en describir e interpretar las percepciones y creencias construidas por los docentes de la enseñanza básica y media en relación con las funciones sociales que asignan a la EF. Concretamente nuestras preguntas iniciales de investigación que nos han servido de guía han sido tres:

¿Cuáles son las creencias de los docentes de EF respecto a la función social que debe aportar el área?

¿Qué barreras a nivel personal, profesional e institucional perciben para orientar la EF hacia un enfoque pedagógico que busque la transformación social y trate de minimizar las desigualdad?

¿Qué soluciones o alternativas ven viables los docentes de EF de incorporar en el área para generar un trabajo transformativo? 


\section{METODOLOGÍA}

Dada la naturaleza de nuestro objeto de estudio, la metodología del mismo se enmarcó en la fenomenología interpretativa (STRAUSS; CORBIN, 2002). La recogida de datos se realizó a través de 3 grupos focales y 12 entrevistas en profundidad de carácter individual. La selección de los participantes para los grupos focales (11 participantes en el primero, 8 en el segundo y 5 en el tercero) y las entrevistas se realizaron a partir de criterios que permitieron representatividad en función de: (a) de diferentes zonas del país [norte, centro y sur], (b) experiencias pedagógicas en la enseñanza primaria y secundaria, (c) sexo, (d) institución donde los participantes se formaron como profesores y profesoras de $\mathrm{EF}^{3}$, y (e) tipo de centro educativo donde lleva a cabo su docen$\mathrm{cia}^{4}$. Tanto las entrevistas como los grupos focales duraron un máximo de 60 minutos.

El análisis de los datos, si bien con carácter fundamentalmente cualitativo, también tuvo una orientación cuantitativa con el fin de mostrar la distribución porcentualmente en el discurso de los participantes y aportar una perspectiva adicional al análisis cualitativo.

Los criterios de selección permitieron, también, realizar el análisis mediante cruces de información con el software informático NVivo 9.0. La primera tarea del análisis consistió en reducir los datos mediante la simplificación, resumen y selección de la información. Además, el análisis se ajustó a los procedimientos de fragmentación y articulación de la "Grounded Theory" (GLASSER; STRAUSS, 1967; STRAUSS; CORBIN, 2002; VALLES, 2007), incluyendo espirales de profundización en las que se iba retrocediendo y avanzando de forma continua a lo largo del proceso hasta construir una teoría lo más coherente posible.

Como señalan Strauss y Corbin (2002, p. 110), la primera codificación de los datos consistió en "el proceso analítico, por medio del cual se identifican los conceptos y se descubren en los datos sus propiedades y dimensiones". En la primera fase, pues, se realizó una lectura analítica de los datos con el fin de seleccionar unidades de significado en base a "criterios temáticos" (RODRÍGUEZ; GIL; GARCÍA, 1999, p.207). En este sentido, se siguió un proceso fundamentalmente inductivo (MILES; HUBERMAN, 1994), aunque, a modo de orientación, se partió de las categorías ya establecidas en los guiones preparados para los grupos focales y las entrevistas individuales.

En la segunda fase se procedió a la síntesis y agrupamiento de unidades de significado en categorías y subcategorías (RODRÍGUEZ; GIL; GARCÍA, 1999). Esta labor de síntesis estuvo orientada a una recreación de lenguaje (RUIZ 2003; STRAUSS; CORBIN, 2002; RODRÍGUEZ; GIL; GARCÍA 1999; MARTÍNEZ, 1999).

Más concretamente, la emergencia de las categorías, desde una primera codificación (creación de nodos libres) hasta llegar a la creación de un árbol de categorías (nodos ramificados), se concretó a través de una indexación inductiva de la lectura de las transcripciones de los grupos focales y las entrevistas. La construcción de los primeros "nodos libres" sirvió de primer borrador para elaborar el árbol de nodos ramificados. En la tabla 1 se presenta la primera codificación.

3 En el contexto de las universidades chilenas es posible percatarse de un grupo de universidades pertenecientes a lo que se dio en llamar el Consejo de Rectores de Universidades Chilenas ( $\mathrm{CRUCH}$ ), grupo al que pertenecen 25 universidades del país, todas creadas previamente a esta desregulación del sistema de creación de instituciones educativas que apareció durante la dictadura militar. Este Consejo es uno de los entes reguladores de estas instituciones. Pero además de estas universidades, podemos encontrar un sinnúmero de universidades de carácter privado a lo largo de todo el país. Esto no quiere decir que todas las universidades pertenecientes al Consejo de Rectores sean públicas, pero sí son instituciones con respaldo estatal y a las que el estado le otorga parte de su financiamiento, a diferencia de las segundas.

4 En Chile existen centros educativos con dependencias administrativas diferenciadas. (i) Centros educativos municipales. (ii) Centros educativos particulares subvencionados. (iii) Centros educativos particulares pagados. 
Tabla 1: Primera codificación: Nodos libres ${ }^{5}$

\begin{tabular}{|c|c|c|c|c|c|c|c|}
\hline $\begin{array}{l}\text { Desvalorización de la } \\
\text { EF }\end{array}$ & 22 & EF y valores & 30 & $\begin{array}{l}\text { Trabajo con la diver- } \\
\text { sidad }\end{array}$ & 2 & $\begin{array}{l}\text { Calificación como pre- } \\
\text { mio o castigo }\end{array}$ & 8 \\
\hline Debilidades EF & 21 & EF y desarrollo integral & 29 & $\begin{array}{l}\text { Reflexión crítica sobre } \\
\text { mitos de la EF }\end{array}$ & 1 & Evaluación tradicional & 6 \\
\hline $\begin{array}{l}\text { Profesor EF como de- } \\
\text { bilidad }\end{array}$ & 20 & EF y transformación??? & 23 & $\begin{array}{l}\text { Respeto a las diferen- } \\
\text { cias }\end{array}$ & 1 & Amenazas & 5 \\
\hline $\begin{array}{l}\text { Barrera EF: cambios } \\
\text { socioculturales }\end{array}$ & 16 & EF e inclusión & 17 & $\begin{array}{l}\text { Tratamiento pedagógi- } \\
\text { co competición }\end{array}$ & 1 & Evaluación diferenciada & 5 \\
\hline Barreras institucionales & 14 & $\begin{array}{l}\text { Importancia de la cons- } \\
\text { trucción de hábitos salu- } \\
\text { dables }\end{array}$ & 15 & $\begin{array}{l}\text { Contextualización en- } \\
\text { señanza }\end{array}$ & 19 & Evaluación como control & 4 \\
\hline Barreras sociales EF & 14 & EF y género & 11 & Trabajo colaborativo & 19 & $\begin{array}{l}\text { Evaluación como res- } \\
\text { paldo }\end{array}$ & 4 \\
\hline $\begin{array}{l}\text { Barreras EF por situa- } \\
\text { ción curricular??? }\end{array}$ & 13 & $\begin{array}{l}\text { Profesionalización do- } \\
\text { cente }\end{array}$ & 11 & $\begin{array}{l}\text { Importancia de la re- } \\
\text { flexión en la labor pe- } \\
\text { dagógica }\end{array}$ & 13 & Evaluación formativa & 4 \\
\hline Barreras profesionales & 13 & $\begin{array}{l}\text { Trabajo con la comuni- } \\
\text { dad }\end{array}$ & 9 & $\begin{array}{l}\text { Diálogo como metodo- } \\
\text { logía de enseñanza }\end{array}$ & 12 & $\begin{array}{l}\text { Relativización califica- } \\
\text { ciones }\end{array}$ & 4 \\
\hline Barreras sociales & 10 & Autonomía profesional & 5 & $\begin{array}{l}\text { Énfasis en la partici- } \\
\text { pación }\end{array}$ & 10 & Evaluación en EF & 3 \\
\hline $\begin{array}{l}\text { Presión social y profe- } \\
\text { sional sobre el profeso- } \\
\text { rado }\end{array}$ & 10 & $\begin{array}{l}\text { EF y desarrollo de va- } \\
\text { lores }\end{array}$ & 5 & $\begin{array}{l}\text { Contextualización en- } \\
\text { señanza a partir de la } \\
\text { reflexión }\end{array}$ & 7 & $\begin{array}{l}\text { Evaluación como pro- } \\
\text { ducto }\end{array}$ & 2 \\
\hline Debilidad familia??? & 8 & Relación afectiva & 5 & $\begin{array}{l}\text { Diálogo y reflexión } \\
\text { como elementos pe- } \\
\text { dagógicos }\end{array}$ & 6 & Autoevaluación & 1 \\
\hline Salud profesional & 6 & $\begin{array}{l}\text { Comprensión para la } \\
\text { autonomía }\end{array}$ & 4 & Autonomía profesional & 5 & $\begin{array}{l}\text { Evaluación como com- } \\
\text { plicación }\end{array}$ & 1 \\
\hline Desgaste profesional & 3 & Diferente no es peor & 4 & $\begin{array}{l}\text { Importancia de la au- } \\
\text { torreflexión }\end{array}$ & 3 & $\begin{array}{l}\text { Evaluación física y acti- } \\
\text { tudinal }\end{array}$ & 1 \\
\hline $\begin{array}{l}\text { Falta de respeto como } \\
\text { barrera }\end{array}$ & 2 & EF y bienestar humano & 4 & $\begin{array}{l}\text { Profesor EF como me- } \\
\text { diador }\end{array}$ & 3 & $\begin{array}{l}\text { Evaluación no es sinóni- } \\
\text { mo de sanción }\end{array}$ & 1 \\
\hline Barrera: EF por género & 2 & $\begin{array}{l}\text { Privado y municipales } \\
\text { similares??? }\end{array}$ & 4 & $\begin{array}{l}\text { Enseñar a través del } \\
\text { juego }\end{array}$ & 2 & Evaluación valores & 1 \\
\hline $\begin{array}{l}\text { Falta de materiales } \\
\text { como barrera }\end{array}$ & 2 & $\begin{array}{l}\text { Crítica al deporte como } \\
\text { eje de la EF }\end{array}$ & 3 & $\begin{array}{l}\text { Importancia del es- } \\
\text { fuerzo }\end{array}$ & 2 & Evaluación y diálogo & 1 \\
\hline Comodidad profesional & 2 & $\begin{array}{l}\text { Importancia de la ense- } \\
\text { ñanza de valores como } \\
\text { experiencia vivida }\end{array}$ & 3 & $\begin{array}{l}\text { Trabajo interdisciplina- } \\
\text { rio y colaborativo }\end{array}$ & 2 & Evaluación y rigor & 1 \\
\hline $\begin{array}{l}\text { Barrera: EF como de- } \\
\text { porte }\end{array}$ & 2 & $\begin{array}{l}\text { Importancia de hacer } \\
\text { conscientes los apren- } \\
\text { dizajes }\end{array}$ & 3 & Trabajo personalizado & 2 & Evaluar para integrar & 1 \\
\hline $\begin{array}{l}\text { Barrera: Escasez de } \\
\text { espacios de diálogo con } \\
\text { los colegas }\end{array}$ & 2 & $\begin{array}{l}\text { Importancia de la auto- } \\
\text { rreflexión }\end{array}$ & 3 & $\begin{array}{l}\text { Deporte como inclu- } \\
\text { sión social }\end{array}$ & 1 & Valorización profesional & 2 \\
\hline $\begin{array}{l}\text { Barrera: EF enseñada por } \\
\text { contenidos tradicionales }\end{array}$ & 1 & $\begin{array}{l}\text { Importancia de lo afec- } \\
\text { tivo }\end{array}$ & 3 & $\begin{array}{l}\text { Educador antes que } \\
\text { profesor }\end{array}$ & 1 & $\begin{array}{l}\text { Trabajar sentido de } \\
\text { EF??? }\end{array}$ & 1 \\
\hline $\begin{array}{l}\text { Debilidad lógica educa- } \\
\text { tiva municipalizado??? }\end{array}$ & 1 & Trabajo familias??? & 3 & EF y no discriminación & 1 & Trabajo interdisciplinario & 1 \\
\hline $\begin{array}{l}\text { Debilidad que al EF no } \\
\text { sea la base del deporte } \\
\text { nacional }\end{array}$ & 1 & $\begin{array}{l}\text { Valoración trabajo estu- } \\
\text { diantes }\end{array}$ & 3 & Énfasis en la reflexión & 1 & Valoración institucional & 1 \\
\hline $\begin{array}{l}\text { Debilidad separación } \\
\text { teoría práctica }\end{array}$ & 1 & $\begin{array}{l}\text { Desarrollo habilidades } \\
\text { sociales }\end{array}$ & 2 & $\begin{array}{l}\text { Enseñanza para la re- } \\
\text { solución de conflictos }\end{array}$ & 1 & $\begin{array}{l}\text { Crítica a políticas minis- } \\
\text { teriales }\end{array}$ & 15 \\
\hline $\begin{array}{l}\text { Hábitos estudiantes } \\
\text { como barrera }\end{array}$ & 1 & $\begin{array}{l}\text { Profesor EF como edu- } \\
\text { cador }\end{array}$ & 2 & $\begin{array}{l}\text { Planificación como faro } \\
\text { que guía la labor edu- } \\
\text { cativa }\end{array}$ & 1 & Críticas al SIMCE ${ }^{6}$ & 12 \\
\hline
\end{tabular}

Fuente: datos de investigación

5 Los números que aparecen en la tabla representan el número de veces que cada código fue referenciado en los datos.

6 Sistema de Medición de la Calidad de la Educación 
A partir de esta primera tabla, emergieron las categorías que se presentan en la tabla 2.

Tabla 2: Categorías emergentes tras la primera codificación: Nodos ramificados.

\begin{tabular}{|c|c|c|}
\hline Categorías (nodos ramificados) & Definición Categorías & Referencias \\
\hline 1. Repitiendo una EF tradicional & $\begin{array}{l}\text { Se hace referencia a características propias de una EF an- } \\
\text { clada en el rendimiento motor, el modelo deportivo compe- } \\
\text { titivo como eje vertebrador de la práctica pedagógica y una } \\
\text { concepción de salud centrada, únicamente, en la dimen- } \\
\text { sión biológica del ser humano. }\end{array}$ & 228 \\
\hline 2. Barreras para una EF crítica & $\begin{array}{l}\text { Los participantes se refieren a las barreras, impedimentos y } \\
\text { dificultades con las que se encuentra la EF para desarrollar } \\
\text { un trabajo pedagógico a partir del cual propender a la trans- } \\
\text { formación y mejora social. }\end{array}$ & 187 \\
\hline 3. Soñando una EF crítica & $\begin{array}{l}\text { Los sujetos se refieren a la posibilidad de construir una EF } \\
\text { alejada de los criterios de rendimiento motor y cuyo interés } \\
\text { central estaría en las posibilidades de aportar a la equidad } \\
\text { social. }\end{array}$ & 206 \\
\hline 4. Cómo hacer una EF crítica & $\begin{array}{l}\text { Aspectos operacionales de la EF crítica que los sujetos } \\
\text { plantean. }\end{array}$ & 111 \\
\hline 5. Evaluando (in) coherentemente & $\begin{array}{l}\text { Relación que existe entre la importancia que le otorgan a } \\
\text { los procesos evaluativos bajo ciertas premisas teóricas/ } \\
\text { epistemológicas y cómo proceden, posteriormente, en el } \\
\text { quehacer docente }\end{array}$ & 53 \\
\hline $\begin{array}{l}\text { 6. Propuestas de superación de } \\
\text { barreras }\end{array}$ & $\begin{array}{l}\text { Procedimientos que los participantes plantean para superar } \\
\text { las barreras ya comentadas anteriormente en la categoría } \\
2 .\end{array}$ & 5 \\
\hline 7. Críticas del profesorado & $\begin{array}{l}\text { Los sujetos consideran una serie de importantes críticas } \\
\text { sobre las que justifican cierta desvalorización docente. }\end{array}$ & 27 \\
\hline
\end{tabular}

Fuente: datos de investigación

La tercera fase consistió fundamentalmente en la contextualización y constatación de los hallazgos alcanzados con otros estudios, para finalmente elaborar un informe narrativo de los resultados y las conclusiones.

\section{RESULTADOS Y DISCUSIÓN}

Para facilitar la compresión de los resultados, se ha divido este apartado en dos partes: resultados cuantitativos y resultados cualitativos.

\subsection{Resultados cuantitativos}

En la gráfica 1 es posible identificar cómo el discurso del profesorado está centrado, con diferente intensidad porcentual, en las siete grandes categorías señaladas con anterioridad. 
Gráfica 1: Presencia porcentual de las categorías de análisis en el discurso de los participantes.

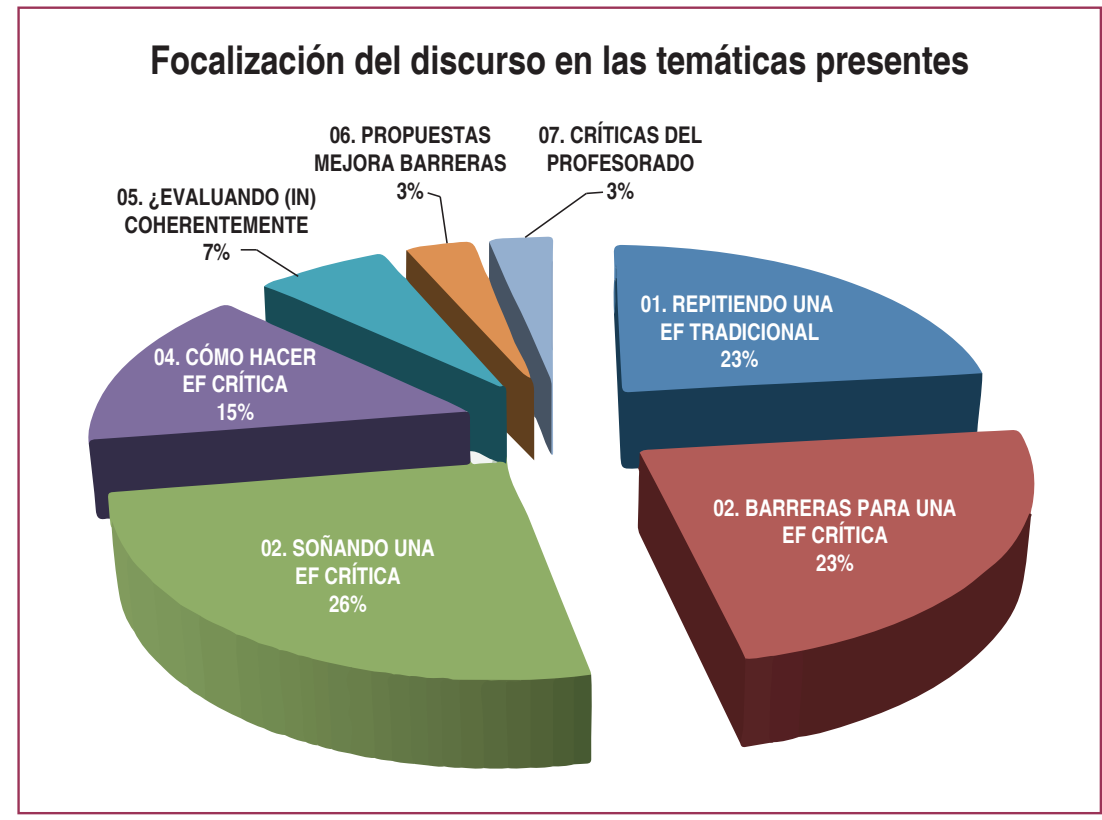

Fuente: Datos de investigación

Ahora bien, el contenido del discurso del profesorado es distinto si nos atenemos a algunas de sus características, como por ejemplo: (a) docentes de primaria o secundaria, (b) tipo de centros educativos donde trabajan, y (c) universidades donde se formaron (Ver gráfica 2).

Las categorías 1, 2, 3 y 5 presentan una intensidad discursiva relativamente similar; sin embargo, al hacer mención al cómo hacer una EF crítica (categoría 4) y al construir críticas sobre las políticas ministeriales (categoría 7), los profesores de enseñanza primaria presentan una mayor intensidad discursiva (ver figura 2). Pareciera que las lógicas menos escolarizadas y escolarizantes (más holísticas y complejas) de la etapa de enseñanza primaria del sistema escolar están influyendo en esta importante diferencia. Esta idea concuerda con otras investigaciones realizadas en Chile (CALVO, 2012).

Gráfica 2: Comparativa porcentual de la intensidad del discurso del profesorado según el nivel educativo en el que se imparte la docencia.

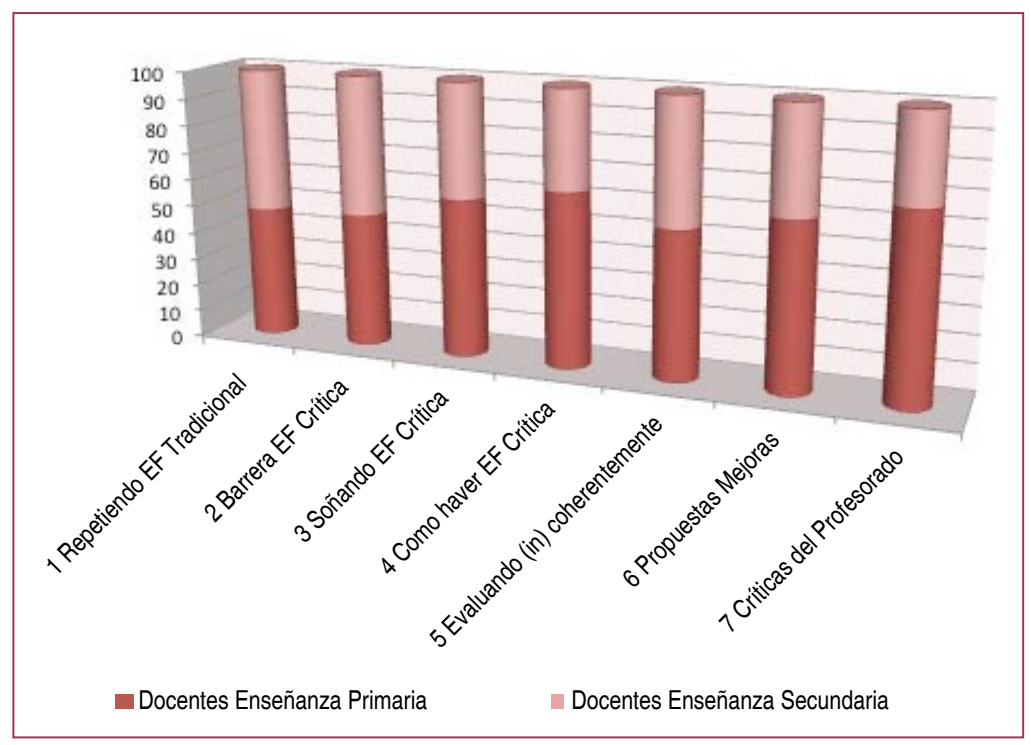


La intensidad del discurso es también diferente si comparamos al profesorado que trabaja en centros educativos privados, subvencionados o municipales (ver gráfica 3). En estos últimos, los docentes perciben que las funciones más importantes de la EF están centradas en la construcción de una orientación crítica a la EF tradicional, ya que esta última está más centrada en reproducir estereotipos corporales y de un concepto de salud entendido como ausencia de enfermedad más que como bienestar humano en todas sus dimensiones. Lo contrario ocurre con los docentes de los centros privados y subvencionados. Es posible percibir, también, que la categoría 7 "Crítica de los docentes" sigue un mismo patrón. La situación de diversidad económica, social y psicológica con la que trabajan los profesores de EF en la enseñanza municipal podría explicar estas diferencias (MORENO, 2011).

Gráfica 3: Intensidad porcentual del discurso del profesorado según tipos de centros educativos en los que imparte docencia.

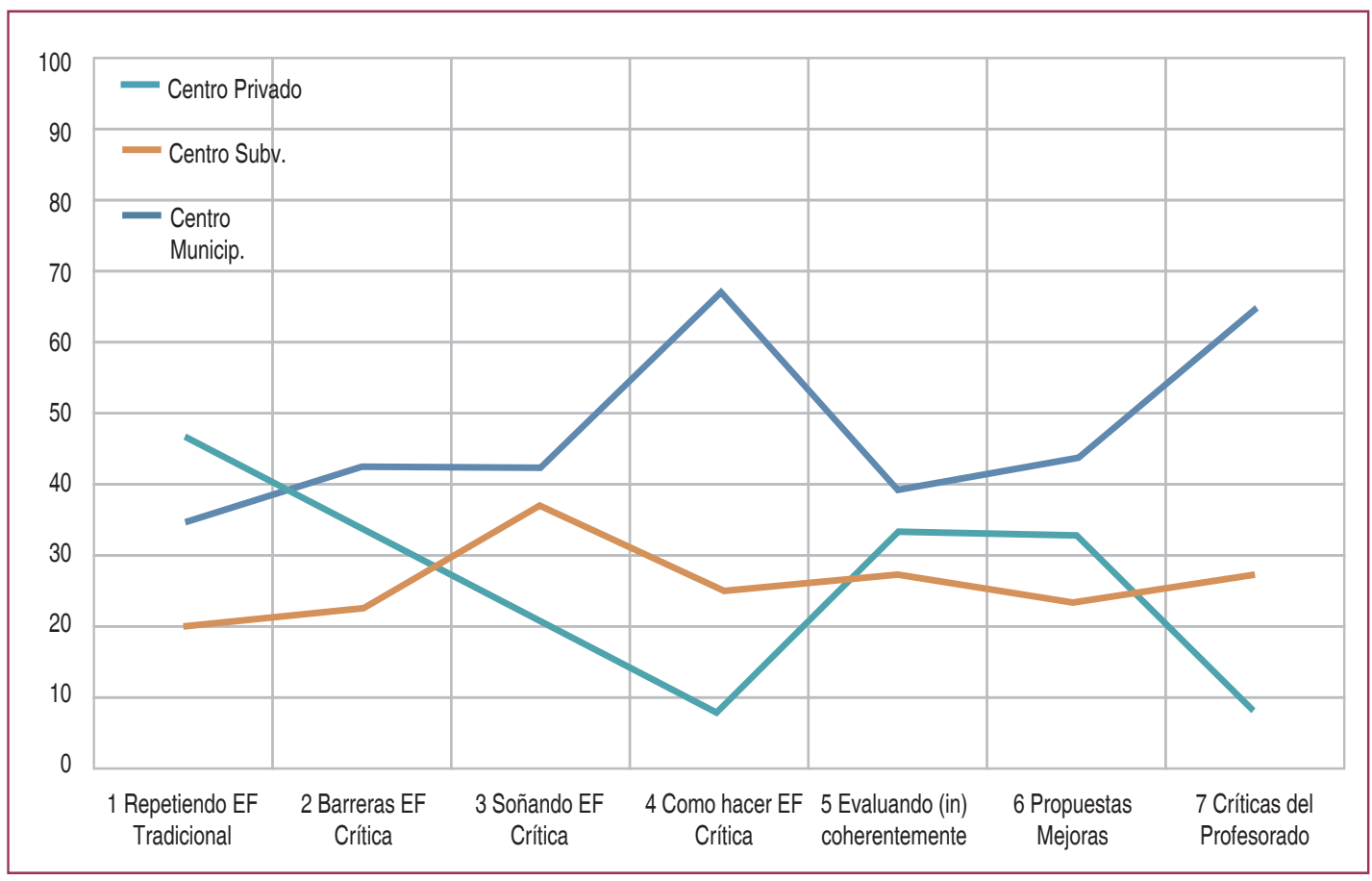

Fuente: datos de investigación

Algo similar ocurre con el discurso de los profesores en relación con el tipo de institución en la que se formaron (ver gráfica 4). En general, es posible percibir que quienes estudiaron en Universidades del Consejo de Rectores, a diferencia de los que no lo hicieron, pretenden construir una EF más allá de una perspectiva tradicional, poniéndo énfasis en la investigación y el pensamiento crítico. Esto quizás pueda ser explicado por el hecho de que universidades que forman parte de este conglomerado reciben la mayor parte de los fondos del Estado, a la vez que tienen como objetivos principales focalizar sus tareas, además de en la docencia, en las áreas de investigación-innovación y extensión; mientras que las no pertenecientes a este conglomerado tienen un enfoque meramente pedagógico. Además, las primeras poseen un gran número de académicos a jornada completa, mientras que en las segundas abundan los profesores contratados por horas (OCDE, 2009). 
Gráfica 4: Intensidad porcentual del discurso según el tipo de centro universitario donde se formaron los participantes.

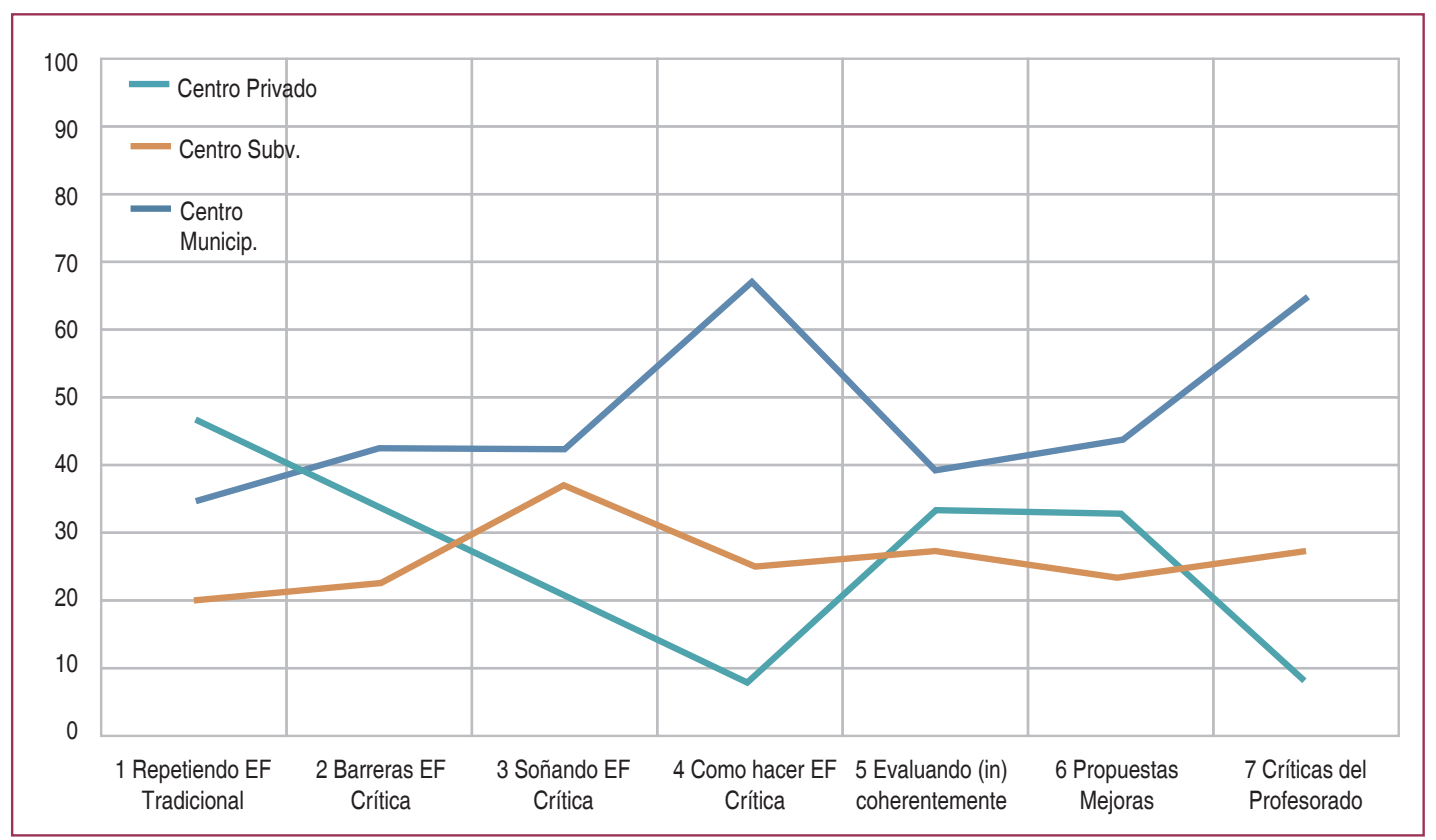

Fuente: datos de investigación

\subsection{Resultados Cualitativos}

Pasamos ahora a desarrollar la mirada cualitativa de nuestro trabajo, intentando responder a cada uno de los objetivos específicos de nuestra investigación.

\subsubsection{Creencias del profesorado con respecto a la EF}

A este respecto, es interesante observar como las creencias del profesorado en relación con las funciones de la EF escolar muestran una relación dicotómica para algunos y complementaria para otros, entre una EF que reproduce una perspectiva tradicional ligada al deporte, el desarrollo de la condición física y ciertos estereotipos sociales; y otra visión que entiende que la EF puede ser útil para el desarrollo de ciertas competencias que ayuden a comprender y transformar las desigualdades sociales del alumnado. A continuación mostraremos la caracterización de las dos creencias sobre la EF como asignatura del currículum escolar.

\subsubsection{Repitiendo una EF tradicional}

Con relación a las percepciones que centran la función principal de la EF tradicional, encontramos 3 aspectos destacados: (a) formación deportiva y desarrollo de la condición física y la salud, (b) repetición acrítica de estereotipos asociados a la EF, y (c) EF como vía de distracción de las circunstancias y problemas personales del alumnado.

a) Formación deportiva y desarrollo de la condición física y la salud

Los docentes hacen alusión, constantemente, al deporte, la condición física y a un 
mayor y mejor estado de salud como contenidos centrales de la asignatura. Esta percepción parte de la creencia de que el deporte es sinónimo de mejora de la condición física y que ésta equivale a un mejor bienestar. Sin embargo, esta creencia contradice evidencia científica que, desde hace décadas, enfatiza que es una lógica equivocada, sobre en todo en niños y niñas, pues, en esas edades, los niveles de condición física están determinados por factores genéticos y de maduración, más que por hábitos de actividad física (FOX 1991).

La presencia constante del deporte, como contenido casi exclusivo de la EF escolar, es posible visualizarla en las propias palabras del profesorado. En uno de los grupos focales (GF1) se hace mención explícita a que "[...] el objetivo personal que tenemos todos los profesores de EF es la formación en el área del deporte."

Esta idea también se refleja a nivel particular entre los participantes. Véase estos dos ejemplos:

De quinto a segundo de secundaria es un día acondicionamiento y el otro de deportes, y en tercero y cuarto como tienen una vez a la semana este año es solamente deporte, no hay acondicionamiento [...], y de primero a cuarto básico se hace deporte los dos días a la semana. (Adolphe)

Elvira comenta que "[...] la única forma que tú [sic] mueves a los chicos en el colegio es con deporte". A su vez, la preocupación con el desarrollo de la salud y, más concretamente, en relación a la lucha contra la obesidad, se hace patente en otro de los grupos de discusión:

Hoy en día también hay un problema general y grandote que es la obesidad, entonces nosotros le vamos a mostrar todos los deportes para que después en la vida cotidiana de ellos puedan rescatar uno de toda esa gama de deportes, por algo tenemos atletismo, basquetbol, fútbol, no sé tenemos todos los deportes y eso es nuestro trabajo. Esta relación entre deporte y salud queda aún más clara si rescatamos las palabras de Barack, al decirnos que [...] la actividad física te da más posibilidades si centras la EF en el rendimiento motor a través de la utilización de tu cuerpo. (GF2)

b) Repetición acrítica de estereotipos asociados a la EF tradicional

El primer estereotipo desarrollado por varios participantes está relacionado con entender que la EF puede ayudar en la formación deportiva y que podría posibilitar que algún estudiante llegara a ser deportista profesional. Evans, por ejemplo, nos señala que "[...] yo siempre les doy ejemplos de deportistas que hoy día son famosos y a [los alumnos] les motiva mucho llegar en algún momento a ser eso. Yo les enseño que pueden perfectamente llegar a ser eso [...]"

El segundo estereotipo se focaliza en la relación acrítica entre la realización de actividad física y la adquisición de ciertos valores que, en última instancia pueden conducir a la superación de la pobreza. "Sí, es súper importante para la parte social el desarrollo de las habilidades que se desarrollan [a través de] la actividad física y el deporte. El espíritu de superación [que puede llevar a] salir de la pobreza es súper importante" (Elvira).

Por último encontramos la presencia de estereotipos de género, lo que dificulta un trabajo pedagógico coeducativo en las clases de EF. Alison considera "[...] que las niñas deben trabajar a parte de los hombres" pues deben "desarrollar otro tipo de cosas". 
c) EF como una vía de distracción de las circunstancias y problemas personales del alumnado

Un tema recurrente entre los participantes es la conceptualización de la EF como vía de distracción de todas las problemáticas sociales, familiares y personales que puede estar experimentado el alumnado (MORENO et al. 2013). "La sesión física sirve para que los chicos puedan divertirse y puedan compartir en ese momento y olvidarse de su situación social. No sé, pero creo que se desarrollan habilidades sociales bastante positivas" (GF2). En este sentido, podría decirse que la EF se centra más en una intencionalidad puramente recreativa que educativa. Alison, por ejemplo, nos señala que ve la EF "[...] como recreación más que nada, liberación, sobre todo, en los sectores de bajos recursos [económicos]".

\subsubsection{Aspiración a una EF crítica}

La finalidad, desde una perspectiva crítica, sería "formar personas" (GF3) a través no de la repetición de ciertos gestos motores sino de la construcción de hábitos saludables en todas las dimensiones del ser humano.

Más concretamente, Jimmy señala la importancia de la EF para el desarrollo de valores como la aceptación de la diversidad.

Trabajar en equipos, respetar al que está al lado, respetar las personas que son diferentes en cuanto a niveles por ejemplo. Los alumnos, a veces discriminan, cuando forman grupos, siempre dejan de cierta forma al gordito al final. [El profesor debe hacer] que esas personas se sientan aceptadas en un grupo.

Arnaud, por su parte, enfatiza "[...] la formación social a través de la EF" y, más concretamente, a partir "del trabajo en grupo", con la intención de que "aparezcan líderes, y ellos también reconozcan situaciones del contexto donde no siempre van a ser escuchados, no siempre. [...] su opinión va a ser la que adquiera mayor validez; sino que [lo importante es la] la construcción que ellos generen a partir de este trabajo de grupo." Cornelio pone el énfasis en el "valor humano en cuanto al desarrollo de valores" que puede tener la EF escolar. "No tienen que golpearse por una pelota, no tienen que agredirse; pueden compartir, pueden desarrollar valores, pueden hacer amigos. No es necesario que tengan que llegar a la grosería o a la violencia verbal, gestual o postural."

Para ello hay quien propone una EF alejada del deporte y el desarrollo de la condición física, es decir, se aboga por una asignatura más coherente con un desarrollo integral de la persona que "[...] contribuya a la formación humana en toda su complejidad" (Luke). También se concibe una EF que permita generar, "una instancia donde todos los alumnos se puedan integrar y donde no sólo se desarrolle el movimiento, sino que se permita construir todo tipos de habilidades sociales para convertirse en un ciudadano crítico" (Axel).

Evans comenta que la EF:

[...] puede y debe dirigirse a encaminar y enderezar el camino de los alumnos de sectores vulnerables, a transformar la situación desigual en la que se encuentran. Ello podría conseguirse si se logra [...] trascender los aprendizajes generados en EF (Arnaud). Esto se conseguirá, según el mismo Arnaud, si [...] la asignatura les ayuda a lograr muchas cosas, a trascender socialmente. 
Cathy señala que los "[...] centros educativos y las asignaturas presentes en ellos son la base para cambiar el mundo. Si yo quiero cambiar mundo no saco nada con cambiar a los adultos, a los que tengo que cambiar es a los niños, desde ahí parto formando el futuro de Chile."

\subsubsection{Barreras para la transformación}

Las barreras que los participantes señalan para la puesta en práctica real de una EF crítica y constructora de mayor equidad y justicia social son cuatro:

i. Barreras por cambios socioculturales: En este sentido, se hace referencia a la complejidad propia de la asignatura en un mundo que ha cambiado excesivamente y que ha provocado que "[...] las enfermedades de la tercera edad aparezcan en la niñez" (Luke), lo que provoca que "trabajemos con niños y jóvenes en una situación para la cual no fuimos formados" (GF2).

ii. Barreras por la situación curricular de la EF: Aquí se plantean las incoherencias de una lógica curricular que, por un lado, dice poseer una intencionalidad educativa mientras que, por otro lado, se ajusta a, y perpetúa, un sistema de medición de la calidad (SIMCE) centrado en otras intencionalidades, lo que termina constituyendo una barrera para el desarrollo educativo de la EF como asignatura del currículum escolar. "EI SIMCE no mide nada, no mide el estado de salud de un alumno, al contrario, lo inhabilita o lo perjudica. Hicieron el SIMCE y varios alumnos salieron con lesiones lumbares y abdominales" (Jimmy).

iii. Barreras de formación profesional: Se incluyen aquí las referencias a la débil formación que hoy día está recibiendo el profesorado de EF, lo que termina provocando que los mismos docentes no sean capaces de construir una asignatura más ligada al ámbito educativo y no relacionada, únicamente, con la práctica deportiva. Lo mismo ocurre con la formación continua del profesorado. "Es muy importante que nos podamos capacitar. Yo trabajo en un colegio municipal y la capacitación es bastante escasa, los tiempos de capacitación bastante escasos y, en general, las horas de trabajo son muchas. Un profesor que tenga 44 horas va a trabajar 60 y va a llegar a su casa; ¿va a seguir trabajando?" (Evans).

iv. Desvalorización social de la EF escolar. Es "triste llegar a final de año frustrado. Pasó el año, y me encuentro sometido por un sistema escolar que no me representa en nada [...]. Los apoderados se oponen a mi criterio, el jefe no me apoya y me pide que cambie la planificación [...]. Ello porque la EF no es una disciplina fuerte que no se valora socialmente" (Cornelio).

3.2.1.4 Escasas soluciones y alternativas que los profesionales del área identifican para un trabajo transformativo en la EF escolar

Las propuestas que los participantes dan con relación a cómo conseguir que la EF sea una asignatura que persiga un trabajo pedagógico orientado a la transformación de las desigualdades sociales están relacionadas con tres aspectos concretos:

i. Contratar profesorado especialista de la EF en todos los centros, en todos los niveles del sistema educativo. "[...] que haya profesores que sean especialistas desde preescolar hasta cuarto medio" (Adolphe).

ii. Exigir más horas para la asignatura: "que sea obligatorio para todos los centros y [...] que el tema de las cuatro horas dos veces a la semana también sea obligatorio" (Adolphe). 
iii. Incentivar al alumnado para que procure concienciar a padres y madres para la adopción de estilos de vida saludables a nivel familiar: "Incentivar a los alumnos que motiven o estimulen a sus mismos papás a realizar actividad física" (Jimmy).

\section{CONCLUSIONES}

Queremos concluir este trabajo enfatizando que existe una relación dicotómica para algunos docentes, complementaria para otros profesionales, entre una EF que reproduce una perspectiva tradicional ligada al deporte, el desarrollo de la condición física y ciertos estereotipos sociales; y otra visión que entiende que la EF debe estar al servicio del desarrollo de competencias de comprensión y transformación de las desigualdades sociales que experimenta el alumnado. Con relación a las creencias que centran la función principal de la EF en una perspectiva tradicional, encontramos 3 aspectos destacados: (a) formación deportiva y desarrollo de la condición física y la salud, (b) repetición acrítica de estereotipos asociados a la EF, y (c) EF como vía de distracción de las circunstancias y problemas personales del alumnado. En cuanto a las creencias relacionadas con una EF dirigida hacia el trabajo por las desigualdades sociales, mostramos cierta aspiración a un EF crítica, aunque muy superficial y poco fundamentada. Se enuncian barreras importantes para este trabajo, a saber: cambios socioculturales; situación curricular de la EF; formación profesional y desvalorización social de la EF escolar.

\section{REFERENCIAS}

BALL, Stephen. Education for sale! The commodification of everything. London: University of London, 2004.

BARBERO, José. El darwinismo social como clave constitutiva del campo de la actividad física educativa, recreativa y deportiva. Revista de Educación, Madrid, n. 359, p. 580-603, 2012.

BONETA, Martí. Un mundo que se mueve con el aprendizaje servicio. Tándem: Didáctica de la Educación Física, Barcelona, n. 44, p. 57-61, 2014.

CALVO, Carlos. Del mapa escolar al territorio educativo: disoñando la escuela desde la educación. La Serena: Universidad de La Serena, 2012.

CERVANTES, Carlos; MEANEY, Karen. Examining Service-Learning Literature in Physical Education Teacher Education: Recommendations for Practice and Research. Quest, Atlanta, v. 65, n. 3, p. 332353, 2013.

CHIVA, Oscar; GIL, Jesús; HERNANDO, Carlos. Innovación metodológica en la universidad Aprendizaje servicio en la didáctica de la expresión corporal y los juegos motrices. Tándem: Didáctica de la Educación Física, n. 44, p. 41-48, 2014.

DEVÍS, José. La investigación sociocrítica en la educación física. Estudios Pedagógicos, v. 38, n. 1 , p. 125-153, 2012.

DEVÍS, José. Actividad física, deporte y salud. Barcelona: Inde, 2000.

DEVÍS, José. Socially Critical Research Perspectives in Physical Education. En: KIRK, David; O'SULLIVAN, Mary; MACDONALD, Doune. (Edit.). Handbook of physical education. London: Sage, 2006. p. 37-59.

DUBET, Francios. Repensar la justicia social: Contra el mito de la igualdad de oportunidades. 
LORENTE, Eloisa; JOVEN, Alfredo. Autogestión en Educación Física: una investigación etnográfica. Cultura y Educación, v. 21, n. 1, p. 67-79, 2009.

EVANS, John. Physical Education as porn! Physical Education and Sport Pedagogy, Worcester, v.18, n.1, p. 75-89, 2013.

FOX, Ken. Motivating children for physical activity: towards a healthier future. Journal of Physical Education, Recreation and Dance, Reston, v. 62, n. 7, p. 34-38, 1991.

GARDNER, Howard. Estructuras de la mente: la teoría de las inteligencias múltiples. Madrid: Fondo de Cultura Económica, 1994.

GARDNER, Howard. Inteligencias múltiples: la teoría en la práctica. Barcelona: Paidós, 2005.

GLASER, Barney; STRAUSS, Anselm. The discovery of grounded theory: Strategies for qualitative research. Chicago: Aldine, 1967.

GOLEMAN, Daniel. Inteligencia emocional. Barcelona: Kairós, 1996.

HARWOOD, Valerie. Tehorizing Biopedagogies. En: WRIGHT, Jean; HARWOOD, Valerie. Biopolitics and the "Obesity Epidemic". Goberning Bodies. New York: Routledge, 2009. p. 15-30.

HUXLEY, Aldous. Un mundo feliz. Barcelona: Debolsillo, 2001.

LEMOS, Lovane; VERONEZ, Luiz; MORSCHBACHER, Marcia; BOTH, Vilmar. As contradições do processo de elaboração das diretrizes curriculares nacionais dos cursos de formação em educação física e os movimentos de resistência à submissão ao mercado. Movimento, Porto Alegre, v. 18, n. 3 , p. $27-49,2012$

LORENTE, Eloisa; JOVEN, Alfredo. Autogestión en Educación Física: una investigación etnográfica. Cultura y Educación, Barcelona, v. 21, n. 1, p. 67-79, 2009.

LORENTE, Eloisa; KIRK, David. The case for democratic assessment practices within a critical pedagogy of physical education teacher education. European Physical Education Review, Chester, v. 20, n. 1, p. 104-119, 2014

MARTÍNEZ, Miguel. La investigación cualitativa etnográfica en educación: Manual teóricopráctico. México: Trillas, 1999.

MILES, Michael; HUBERMAN, Matthew. Qualitative data analysis: an expanded sorcebook. Newbury Park: Sage, 1994.

MORENO, Alberto; TRIGUEROS, Carmen; RIVERA, Enrique. Percepciones sobre la autoevaluación en la formación de Profesores de Educación Física. Revista Internacional de Medicina y Ciencias de la Actividad Física y del Deporte, Madrid, v. 13, n. 52, p. 719-735, 2013.

MORENO, Alberto et. al. La educación física chilena. Un modelo tecnocrático de la enseñanza y desvalorización del colectivo docente. Tándem: Didáctica de la Educación Física, Barcelona, n. 42, p. 7-17, 2013.

MORENO, Alberto. Percepciones del profesorado universitario en relación a la función de transformación de la educación física como asignatura de curriculum escolar: el caso de Chile. 2011. Tesis (Doctoral) - Granada: Universidad de Granada, 2011.

OCDE. Informe Revisión de Políticas Nacionales de Educación. La Educación Superior en Chile, 2009. Disponible en: <http://feuc.cl/wp-content/uploads/2011/07/ Informe-OCDE-ES-en-Chile.pdf>. Acceso en: 11 ago. 2010.

ORWELL, George. 1984. Barcelona: Destino Libro, 2003.

PÉREZ, Ángel; SOTO, Encarnación. Luces y sombras de PISA. Sentido educativo de las evaluaciones externas. Cultura y Educación, Barcelona, v. 23, n. 2, p. 171-182, 2011.

PRAT, María; SOLER; Susanna. El espectáculo deportivo, ¿un contexto educativo?: análisis de las 
actitudes de los adolescentes como espectadores. Cultura y Educación, Barcelona, v. 21, n. 1, p. 43-54.

RODRÍGUEZ, Gregorio; GIL, Javier; GARCÍA, Eduardo. Metodología de la investigación cualitativa. Málaga: Aljibe, 1999.

RUBIO, Laura; CAMPO, Laura; SEBASTIANI, Enric. Educación Física y Aprendizaje Servicio. Tándem: Didáctica de la Educación Física, Barcelona, n. 44, p. 7-14, 2014.

RUIZ, José Ignacio. Metodología de la investigación cualitativa. Bilbao: Universidad de Deusto, 2003.

SICILIA, Álvaro; FERNÁNDEZ, Juan. Reflecting on the moral bases of critical pedagogy in PETE: toward a Foucaultian perspective on ethics and the care of the self. Sport, Education and Society, London, v. 14, n. 4, p. 443-463, 2009.

SPAAIJ, Ramón; JEANES, Ruth. Education for social change? A Freirean critique of sport for development and peace. Physical Education and Sport Pedagogy, Worcester, v. 18, n. 4, p. 442457, 2013.

STRAUSS, Anselm; CORBIN, Juliet. Bases de la investigación cualitativa: Técnicas y procedimientos para desarrollar la teoría fundamentada. Colombia: Universidad de Antioquia, 2002.

SOLER, Susanna. Los procesos de reproducción, resistencia y cambio de las relaciones tradicionales de género en la Educación Física: el caso del fútbol. Cultura y Educación, Barcelona, v. 21, n. 1, p. 31-42, 2009.

TIRADO, Miguel; VENTURA, Carles. Propuestas para el desarrollo de la competencia crítica en el alumnado de Educación Física. Cultura y Educación, Barcelona, v. 21, n. 1, p. 55-66, 2009.

VALLES, Miguel. Técnicas cualitativas de investigación social: Reflexión metodológica y práctica profesional. Madrid: Síntesis, 2007.

VICENTE, Miguel. Crítica de la educación física y Educación Física Crítica en España. Estado (crítico) de la cuestión. Movimento, Porto Alegre, v. 19, n. 1, p. 309-329, 2013.

\section{Dirección Postal}

Pontificia Universidad Católica de Valparaíso

Facultad de Filosofía y Educación

Escuela de Educación Física

Grupo de Estudio "Motricidad y Educación"

Avenida El Bosque, 1290 - Santa Inés - Viña del Mar - V Región Chile

Financiación: Proyecto Investigación Fondecyt 11110016, financiado por Fondo Nacional de Investigación Científica y Tecnológica de Chile. 